\title{
Neonatal Septicemia in Al-Jahra Hospital, Kuwait: Etiologic Agents and Antibiotic Sensitivity Patterns
}

\author{
Huda H.A. Gomaa ${ }^{a}$ Edet E. Udo ${ }^{c}$ Usha Rajaram ${ }^{b}$ \\ Departments of a Microbiology and ${ }^{b}$ Neonatology, Al-Jahra Hospital, and ${ }^{c}$ Department of Microbiology, \\ Faculty of Medicine, Kuwait University, Kuwait
}

\section{Key Words}

Neonatal septicemia $\cdot$ Antibiotic resistance $\cdot$ Neonatal sepsis

\begin{abstract}
Background: Neonatal septicemia (NNS) occurs frequently in neonatal intensive care units (NICU) and is often associated with high morbidity and mortality. However, information on its incidence and causative agents in Kuwait hospitals is scanty. Objectives: To investigate the bacterial causative agents of NNS in a NICU and their susceptibility patterns to antimicrobial agents. Methods: Between May 1 and December 31, 1996, blood cultures were performed on all admissions to the Neonatal Unit, Al-Jahra Hospital, Kuwait, with the Bactec 9240 instrument (Becton Dickinson, USA). Microorganisms were identified by cultural characteristics, Gram stain and biochemical profiles and antimicrobial susceptibility patterns performed by disk diffusion and by measuring their minimum inhibitory concentrations. Results: From a total of 995 neonates admitted to the neonatal unit during the study period, $117(11.7 \%)$ had positive blood cultures. Eighty-seven (8.7\%) of the neonates had confirmed septicemia. Gram-positive organisms were cul-
\end{abstract}

tured from 65 (75\%) and gram-negative organisms from $22(25 \%)$ of them. The most frequent organisms isolated were Staphylococcus epidermidis (34\%), Streptococcus viridans (28\%) and Candida species (14\%). Resistance to ampicillin and cephalosporins was detected in both gram-positive and gram-negative organisms associated with sepsis. Conclusions: The study identified the common bacterial pathogens associated with NNS in a neonatal unit, their susceptibility patterns to antimicrobial agents and emphasized the importance of understanding local epidemiology of NNS in formulating an antibiotic policy.

\section{Introduction}

Neonatal septicemia (NNS) continues to be a major problem for neonates in neonatal intensive care units (NICUs) around the world [1-7] with the incidence in the USA varying from 5 to $32 \%$ [1]. NNS is an important cause of morbidity and mortality in the early days of life despite major advances in neonatal care and a cause of stress not only to the neonates but also to the parents and hospital staff $[2,3]$.

\section{KARGER \\ Fax +4161306 1234 \\ E-Mail karger@karger.ch \\ www. karger.com}

Dr. Edet E. Udo

Department of Microbiology, Faculty of Medicine

Kuwait University, PO Box 24923

13110 Safat (Kuwait)

Tel./Fax +965 533 2719, E-Mail edet@hsc.kuniv.edu.kw 
NNS is caused by a variety of gram-positive as well as gram-negative bacteria and sometimes yeast. However, institutional as well as regional differences in the types of pathogens and severity of NNS have been demonstrated $[1,2,8-10]$. Differences in causative organisms have been noted between early- and late-onset septicemias [3, 6]. Furthermore, the epidemiology of neonatal septicemia within the same geographical locations may change with time [7, 11, 12]. Several reports have implicated commensal species found on the skin and sometimes in the intestine of the neonates, such as coagulase-negative staphylococci and Candida albicans, as a significant cause of neonatal infections.

The increasing incidence of NNS in NICUs has been attributed to the widespread use of invasive procedures such as central venous catheters, intravenous nutrition, overcrowding and staff shortage [13-19]. Therefore, it is necessary to have a continuing bacteriological surveillance of NICUs so as to be aware of the common pathogens that cause septicemia, and their susceptibility to commonly used antimicrobial agents. This knowledge should guide clinicians on the appropriate use of such antimicrobial agents. Although studies on the incidence of NNS have been conducted in Saudi Arabia [7], information on its incidence in Kuwait hospitals is scanty [20,21] and is lacking in Al-Jahra Hospital. The purpose of this study, therefore, was to investigate the incidence of NNS, their causative microbial agents in Al-Jahra Hospital in Kuwait and to determine the antimicrobial sensitivity patterns of such pathogens. The Al-Jahra Hospital is a 420-bed general hospital that serves mostly the Beduin tribe. The hospital consists of general medical, urology, pediatrics, surgical, dialysis, intensive care unit, and outpatient departments.

\section{Patients and Methods}

\section{Patients}

All neonates admitted to the neonatal unit of Al-Jahra Hospital, Kuwait from May 1 to December 31, 1996 who developed septicemia were studied prospectively. For inclusion in the study the following criteria had to be met: (i) clinical evidence suggestive of sepsis, e.g. lethargy, feed intolerance, unexplained metabolic acidosis, unexplained respiratory distress, jaundice, instability of thermoregulation or glucose homeostasis, apnea, bradycardia, mottling of skin and hypotonia [12]; (ii) hematologic evidence of sepsis, namely thrombocytopenia, neutropenia or an elevated fraction of immature to total neutrophils using the criteria of Manroe et al. [22]; (iii) a diagnosis of NNS confirmed by a positive blood culture for a single organism. Multiple positive blood cultures in the same infant, which grew the same species with the same antibiotic susceptibilities, were consid- ered as a single case. After samples for the relevant investigation had been obtained, each infant suspected of having septicemia received a combination of antibiotics. Initial antibiotics were a combination of ampicillin and gentamicin or cefuroxime. This initial therapy was modified when culture results and their sensitivity patterns were available.

\section{Isolation of Bacterial Pathogens}

Blood for culture was obtained by venipuncture after preparation of the skin with $70 \%$ isopropyl alcohol or from umbilical catheters using aseptic techniques. Pediatric blood culture bottle (Bactec Peds plus/F 40-ml culture vial, aerobic resin) was inoculated with $1-3 \mathrm{ml}$ of blood. The bottles were incubated in the Bactec 9240 instrument (Becton Dickinson, USA) for at least 7 days. For positive samples, a direct Gram smear was performed for preliminary diagnosis. It was subcultured onto blood agar, MacConkey agar, chocolate agar and Sabouraud's agar for the isolation of Candida species. Identification of the organisms was based on cultural characteristics, results of catalase, coagulase, oxidase, urease, germ tube, optochin, bacitracin and biochemical profiles using Vitek GNI, GPI and YBC cards for gram-negative, gram-positive and yeast identification, respectively (bioMérieux Sa, Marcy-l'Étoile, France) or API tests specific for gram-negative and gram-positive organisms (bioMérieux Sa).

\section{Antibiotic Susceptibility Testing}

Antibiotic sensitivity testing was performed by the disk diffusion method [23] on Mueller-Hinton (MH) agar for rapidly growing organisms and incubated at $35^{\circ} \mathrm{C}$ for $18 \mathrm{~h}$. Methicillin resistance was detected on $\mathrm{MH}$ agar supplemented with $2 \% \mathrm{NaCl}$ incubated for $24 \mathrm{~h}$ at $35^{\circ} \mathrm{C}$. Susceptibility testing for Streptococcus pneumoniae was performed on $\mathrm{MH}$ agar supplemented with $5 \%$ sheep blood. The minimum inhibitory concentration was determined with the Vitek automated machine using GNS and GPS cards for gram-negative and gram-positive sensitivity, respectively (bioMérieux Sa). The following antibiotics were tested for both gram-positive and gram-negative organisms: ampicillin, amoxycillin/clavulanic acid, cephalothin, cefotaxime, ceftazidime, ciprofloxacin, clindamycin, gentamicin, trimethoprim/sulfamethoxazole, amikacin, chloramphenicol. In addition, penicillin $\mathrm{G}$, erythromycin, oxacillin, vancomycin, teicoplanin, were tested against gram-positive organisms. Aztreonam, imipenem, mezlocillin, piperacillin, ticarcillin, tobramycin and ceftriaxone were tested against gram-negative bacilli.

\section{$\beta$-Lactamase Production}

The isolates were tested for the production of $\beta$-lactamases using nitrocefin (Oxoid, Basingstoke, England) according to the manufacturer's instructions.

\section{Results}

\section{Microbial Isolates}

Between May 1 and December 31, 1996, a total of 995 infants admitted to the Neonatal Unit of Al-Jahra Hospital were investigated for bacteremia. One hundred and seventeen (11.8\%) of the neonates had positive blood cultures that yielded different microorganisms. Eighty-seven 
neonates $(8.7 \%)$ were confirmed to have septicemia. Gram-positive organisms constituted $74.7 \%$ of the total microbial isolates. The different organisms isolated from these neonates are presented in table 1 . The most frequently isolated organisms were methicillin-resistant Staphylococcus epidermidis (30/87), Streptococcus viridans (24/87) and Candida spp. (12/87). There were 4 cases of nonenterococcal group-D streptococci and 3 cases of group-B streptococci (GBS, Streptococcus agalactiae). Two cases of methicillin-resistant Staphylococcus aureus were isolated and 1 case of each of methicillin-sensitive S. aureus and S. pneumoniae. Escherichia coli was the most common gram-negative organism isolated followed

Table 1. Microbial agents isolated from blood culture

\begin{tabular}{lrr}
\hline Organism & $\mathrm{n}$ & $\%$ \\
\hline Methicillin-resistant $S$. epidermidis & 30 & 34 \\
Methicillin-resistant $S$. aureus & 2 & 2 \\
Methicillin-susceptible $S$. aureus & 1 & 1 \\
S. viridans & 24 & 28 \\
Streptococcus group D & 4 & 5 \\
S. agalactiae (group B) & 3 & 3 \\
S. pneumoniae & 1 & 1 \\
Candida spp. & 12 & 14 \\
E. coli & 4 & 5 \\
$P$. aeruginosa & 2 & 2 \\
Pasteurella spp. & 1 & 1 \\
S. marcescens & 1 & 1 \\
Flavobacterium spp. & 1 & 1 \\
Nonfermenting gram-negative bacilli & 1 & 1 \\
\hline Total & 87 & 100 \\
\hline
\end{tabular}

by Pseudomonas spp. Other gram-negative bacilli such as Pasteurella spp., Serratia marcescens, Flavobacterium spp. and nonfermenting gram-negative bacilli were isolated less frequently. Isolates from 30 neonates accounting for $25.6 \%$ of the total microbial isolates were regarded as contaminants based on the Centers for Disease Control (CDC) criteria namely: bacteria and fungi that constitute normal skin and neonatal intestinal flora as contaminants when isolated from blood cultures [24]. Based on the CDC criteria, 9 cases of methicillin-sensitive S. epidermidis, 7 cases of diphtheroids, 1 case each of Erysipelothrix rhusiopathiae and Neisseria sicca and mixed growth of two or more organisms isolated from the remaining 12 cases were considered to be contaminants.

\section{Susceptibility to Antibiotics}

The results of sensitivity testing are summarized in tables 2 and 3. Table 2 shows the antimicrobial sensitivity pattern of gram-positive organisms. All of the staphylococci, including the coagulase-negative staphylococci, produced $\beta$-lactamase, were resistant to penicillin $\mathrm{G}$, but were susceptible to vancomycin and teicoplanin. The streptococcal isolates were susceptible to most of the antibiotics but $S$. viridans, non-Enterococcus group-D streptococci and S. agalactiae showed different degrees of resistance to trimethoprim/sulfamethoxazole, gentamicin and erythromycin. As the antibiotic susceptibility patterns of gram-negative organisms presented in table 3 demonstrated, $E$. coli were susceptible to most of the antibiotics except amoxycillin/clavulanic acid and cephalosporins, mezlocillin, piperacillin, and ticarcillin to which some were resistant. Pseudomonas aeruginosa and $S$. marcescens were resistant to most of the antibiotics including the cephalosporins.

Table 2. Antimicrobial susceptibility of gram-positive isolates (number sensitive to commonly used antimicrobials)

\begin{tabular}{|c|c|c|c|c|c|c|c|c|c|c|c|c|c|c|c|c|c|}
\hline Organism & AMP & $\mathrm{AM} / \mathrm{C}$ & $\mathrm{AM} / \mathrm{S}$ & $\mathrm{CF}$ & CTX & CIP & $\mathrm{CC}$ & $\mathrm{E}$ & PG & SXT & GM & OX & VA & $\mathrm{TP}$ & $\mathrm{AK}$ & $\mathrm{C}$ & $\begin{array}{l}\beta \text {-Lactamase- } \\
\text { positive }\end{array}$ \\
\hline $\operatorname{MRSE}(\mathrm{n}=30)$ & ND & ND & 0 & 0 & 0 & 0 & 13 & 4 & 9 & 14 & 25 & 0 & 30 & 30 & 25 & ND & 30 \\
\hline $\operatorname{MRSA}(\mathrm{n}=2)$ & ND & ND & 0 & 0 & 0 & 0 & 0 & 0 & 0 & 0 & 0 & 0 & 2 & 2 & 0 & ND & 2 \\
\hline $\operatorname{MSSA}(n=1)$ & ND & ND & 1 & 1 & 1 & 1 & 1 & 1 & 0 & 1 & 1 & 1 & 1 & 1 & 1 & ND & 1 \\
\hline S. viridans $(\mathrm{n}=24)$ & 24 & 24 & ND & 24 & 24 & ND & 21 & 21 & 24 & 1 & 0 & ND & 23 & ND & ND & 24 & ND \\
\hline Strept. group D $(n=4)$ & 3 & 4 & ND & 4 & 4 & ND & 4 & 0 & 4 & 1 & 1 & ND & 4 & ND & ND & 4 & ND \\
\hline S. agalactiae $(\mathrm{n}=3)$ & 3 & 3 & ND & 3 & 3 & ND & 3 & 3 & 3 & 2 & 3 & ND & 3 & 3 & ND & 2 & ND \\
\hline S. pneumoniae $(\mathrm{n}=1)$ & 1 & 1 & ND & 1 & 1 & ND & 1 & 1 & 1 & 1 & 1 & ND & 1 & ND & ND & 1 & ND \\
\hline
\end{tabular}

$\mathrm{AMP}=$ Ampicillin; $\mathrm{AM} / \mathrm{C}=$ amoxycillin/clavulanic acid $; \mathrm{AM} / \mathrm{S}=$ ampicillin/sulbactam $; \mathrm{CF}=$ cephalothin; $\mathrm{CTX}=$ cefotaxime;

$\mathrm{CIP}=$ ciprofloxacin; $\mathrm{CC}=$ clindamycin; $\mathrm{E}=$ erythromycin; $\mathrm{PG}=$ penicillin $\mathrm{G} ; \mathrm{SXT}=$ trimethoprim/sulfamethoxazole;

$\mathrm{GM}=$ gentamicin; $\mathrm{OX}=$ oxacillin; $\mathrm{VA}=$ vancomycin; $\mathrm{TP}=$ teicoplanin; $\mathrm{AK}=$ amikacin; $\mathrm{C}=$ chloramphenicol;

MRSE = methicillin-resistant $S$. epidermidis $;$ MRSA = methicillin-resistant $S$. aureus $;$ MSSA = methicillin-sensitive $S$. aureus $;$ Strept. = streptococcus. 
Table 3. Antimicrobial susceptibility patterns of gram-negative isolates (number sensitive to commonly used antimicrobials)

\begin{tabular}{|c|c|c|c|c|c|c|c|c|c|c|c|c|c|c|c|c|c|c|}
\hline Organism & AMP & $\mathrm{AM} / \mathrm{C}$ & $\mathrm{AK}$ & $\mathrm{AZ}$ & $\mathrm{CF}$ & CMX & CTX & CTZ & CIP & $\mathrm{C}$ & GM & IMP & MEZ & PIP & TIC & TOB & SXT & $\mathrm{CRO}$ \\
\hline E. $\operatorname{coli}(\mathrm{n}=4)$ & 1 & 2 & 4 & 4 & 2 & 4 & 4 & 4 & 4 & 4 & 4 & 4 & 1 & 1 & 1 & 4 & 3 & 4 \\
\hline P. aeruginosa $(\mathrm{n}=2)$ & 0 & 0 & 2 & 2 & 0 & 0 & 0 & 2 & 2 & 0 & 2 & 2 & 2 & 2 & 2 & 2 & 0 & 0 \\
\hline Pasteurella spp. $(\mathrm{n}=1)$ & 1 & 1 & 1 & 0 & 1 & 1 & 1 & 1 & 1 & 1 & 1 & 1 & 1 & 1 & 1 & 1 & 1 & 1 \\
\hline Flavobacterium spp. $(\mathrm{n}=1)$ & 0 & 0 & 1 & 1 & 1 & 1 & 1 & 1 & 1 & 0 & 1 & 0 & 1 & 1 & 1 & 0 & 1 & 1 \\
\hline Serratia spp. $(\mathrm{n}=1)$ & 0 & 0 & 0 & 1 & 0 & 0 & 1 & 1 & 1 & 1 & 0 & 1 & 0 & 0 & 0 & 0 & 0 & 1 \\
\hline \multicolumn{19}{|l|}{ Nonfermenting } \\
\hline gram-negative bacilli $(\mathrm{n}=1)$ & 1 & ND & 1 & ND & 1 & ND & 1 & ND & ND & ND & 1 & ND & ND & ND & ND & ND & 0 & ND \\
\hline
\end{tabular}

$\mathrm{AMP}=$ Ampicillin; $\mathrm{AM} / \mathrm{C}=$ amoxycillin $/$ clavulanic acid; $\mathrm{AK}=$ amikacin; $\mathrm{AZ}=$ aztreonam; $\mathrm{CF}=$ cephalothin;

$\mathrm{CMX}=$ cefuroxime; $\mathrm{CTX}=$ cefotaxime; $\mathrm{CTZ}=$ ceftazidime; $\mathrm{CIP}=$ ciprofloxacin $\mathrm{C}=$ chloramphenicol; $\mathrm{GM}=$ gentamicin;

$\mathrm{IMP}=$ imipenem; $\mathrm{MEZ}=$ mezlocillin $; \mathrm{PIP}=$ piperacillin; $\mathrm{TIC}=$ ticarcillin $; \mathrm{TOB}=$ tobramycin; $\mathrm{SXT}=$ trimethoprim $/$ sulfamethoxazole;

$\mathrm{CRO}=$ ceftriaxone.

\section{Discussion}

This study investigated the common pathogens associated with NNS and their susceptibility patterns to antimicrobial agents in a neonatal unit for neonates in a general hospital in Kuwait. The results revealed that $8.7 \%$ of the neonates had positive blood cultures for different microorganisms indicating sepsis. The observed incidence is similar to a $9 \%$ incidence of positive blood cultures among neonates in a Saudi Arabian [25] and 7\% incidence in a Malaysian hospital [19], although Dawodu et al. [7] have reported a lower incidence of NNS in a NICU at another Saudi Arabian hospital. Similarly, Kuruvilla [20] reported an even lower incidence among neonates in a maternity hospital in Kuwait. The differences in the results of this study and the previous study in Kuwait are surprising since the maternity hospital cares for more neonates per year than Al-Jahra Hospital. However, increases in NNS have been linked to increased use of prosthetic divices in hospitals in recent years $[2,9,19]$ or to better methods of detection of organisms in blood with more sensitive equipment such as the Bactec 9240 instrument used in the present study. The Bactec 9240 instrument facilitates the early detection of microorganisms from blood cultures of newborn infants when compared with other blood culture methods [26, 27]. This equipment was not available in Kuwait at the time the previous study was done in 1980 [20].

The isolation of gram-positive organisms, and specifically the isolation of S. epidermidis as the most common gram-positive organism was similar to current findings elsewhere [11, 28]. It contrasts with the findings of a previous study in Kuwait hospital where gram-negative bacteria constituted $90 \%$ of the pathogens [20] and similar studies from Nepal, India, Pakistan, Kenya and Saudi Arabia where gram-negative bacteria were the most common organisms associated with NNS [9, 28-31]. Interestingly, although gram-positive cocci were the most common organisms associated with NNS, methicillin-resistant $S$. aureus was less common in this study. A similar low incidence of methicillin-resistant $S$. aureus was observed in North America [1,32], suggesting that it may not be as important as S. epidermidis as a cause of NNS. S. agalactiae (GBS) also constituted only $3 \%$ of the pathogens in this study similar to the low incidence of GBS reported from blood cultures of neonates in Saudi Arabia $(3 \%)$ [25, 33], Kuwait (5\%) [20] and other developing countries $[19,34]$. In contrast, GBS has been an important cause of NNS in Europe, North America [11, 28, 32] and United Arab Emirates [35].

Although E. coli, isolated in 5\% of cases, and GBS constituted a small fraction of pathogens in this study, both are significant pathogens and share several common characteristics. Both have a tendency to colonize the cervix, and sexual partners of infected women can harbor GBS in their urethra and possibly act as reservoirs in certain societies [20]. Therefore, ethnic and socioeconomic differences may contribute to the varying incidence of GBS infection among neonates in different populations $[9,34$, 36].

Besides gram-positive cocci, Candida species were isolated in a significant number of cases. The detection of candidemia in $14 \%$ of the patients was high and may be attributed to the large number of antibiotics used. It may also reflect the immune status of the neonates.

Results of antibiotic susceptibility testing demonstrated that 3 of the $4 \mathrm{E}$. coli isolates were resistant to ampicillin, suggesting that ampicillin would not be active 
against these organisms. However, since they were susceptible to gentamicin, the combination of ampicillin and gentamicin as empiric therapy for neonatal sepsis was appropriate in our setting since gentamicin would appear to be adequate to combat the gram-negative organisms in this situation. It has been suggested in another study that combining third-generation cephalosporins with amikacin offers the best choice for the initial broad spectrum coverage of microorganisms [9]. The results of the susceptibility testing of the gram-negative bacteria isolated in this study support this suggestion except for $P$. aeruginosa and $S$. marcescens that were resistant to these antibiotics (table 3). These results further support the need to establish and review antibiotic sensitivity patterns of local pathogens regularly for optimal therapy [32]. It is also important to seek alternative forms of treatment besides antibiotics such as the use of hyperimmune anti-S. agalactiae antibody preparations or vaccines and anti-E. coli monoclonal antibodies which have been tested for the treatment and/or prophylaxis of neonatal infections [37].

\section{Conclusion}

This study has revealed an increased incidence of NNS in a general hospital in Kuwait and identified the associated common pathogens. The increase in NNS cases could be due to widespread use of invasive procedures in the neonatal unit or better methods of detection of pathogens. The results emphasized the need for continuous evaluation of local antibiotic sensitivity patterns of pathogens of NNS for the formulation of a rational antibiotic policy.

\section{Acknowledgment}

We would like to thank the staff of the Microbiology Laboratory, Al-Jahra Hospital, for their technical assistance.

\section{References}

1 Gaynes RP, Martone WJ, Culver DH, Emori TG, Horan TC, Banerjee SN, Edwards JR, Jarvis JR, Tolson JS, Henderson TS: Comparison of rates of nosocomial infections in neonatal intensive care units in the United States. National Nosocomial Infections Surveillance System. Am J Med 1991;91:192S-196S.

2 Brodie SB, Sands KE, Gray JE, Parker RA, Goldmann DA, Davis RB, Richardson DK: Occurrence of nosocomial bloodstream infections in six neonatal intensive care units. Pediatr Infect Dis J 2000;19:56-65.

3 Cordero L, Sananes M, Ayers LW: Bloodstream infections in a neonatal intensive care unit: 12 years' experience with an antibiotic control program. Infect Control Hosp Epidemiol 1999;20:242-246.

4 Lin IJ, Chen CH, Chen PY, Wang TM, Chi CS: Nosocomial infection in a neonatal intensive care unit from a view point of national health insurance. Acta Paediatr Taiwan 2000;41:123 128.

5 Nicolls TM, Morgan AS, Morris AJ: Nosocomial bloodstream infection in Auckland Healthcare hospitals. N Z Med J 2000;113:96-98.

6 Kuruvilla KA, Pillai S, Jesudason M, Jana AK: Bacterial profile of sepsis in a neonatal unit in South India. Indian Pediatr 1998;35:851-858.

7 Dawodu A, Al-Umran K, Twun-Danso K: A case control study of neonatal sepsis: Experience from Saudi Arabia. J Trop Pediatr 1997; 43:84-88.
8 Sharma PP, Halder D, Dutta AK, Bhatnagar S, Bali A, Kumari S: Bacteriological profile on neonatal septicemia. Indian Pediatr 1987;24: 1011-1117.

9 Bhutta ZA, Naqvi SH, Muzaffar T, Farooqui BJ: Neonatal sepsis in Pakistan. Acta Paediatr Scand 1991;80:596-601.

10 Mir F, Aman S, Raza Khan S: Presentation and pathogens of neonatal sepsis: A review with a study of 50 cases. J Trop Pediatr 1987;33:131135.

11 Gladstone IM, Ehrenkranz RA, Edberg SC, Baltimore RS: A ten-year review of neonatal sepsis and comparison with the previous fiftyyear experience. Pediatr Infect Dis J 1990;9: 819-825.

12 Maxwell FC, Bourchier D: Neonatal septicemia: A changing picture? N Z Med J 1991; 104:446-447.

13 Noel GJ, O’Loughlin JE, Edelson PJ: Neonatal Staphylococcus epidermidis right-sided endocarditis: Description of five catheterized infants. Pediatrics 1988;82:234-239.

14 Mullet MD, Cook EF, Gallagher R: Nosocomial sepsis in the neonatal intensive care unit. $\mathrm{J}$ Perinatol 1998;18:112-115.

15 Butler KM, Baker CJ: Candida: An increasingly important pathogen in the nursery. Pediatr Clin North Am 1988;35:543-563.

16 Sung L, Ramotar K, Samson LM, Toye B: Bacteremia due to persistent strains of coagulasenegative staphylococci in a neonatal intensivecare unit. Infect Control Hosp Epidemiol 1999; 20:349-351.
17 Fulginiti VA, Ray CG: Body surface cultures in the newborn infant: An exercise in futility, wastefulness and inappropriate practice. Am J Dis Child 1988;142:19-20.

18 Goldmann DA, Durbin WA Jr, Freeman J: Nosocomial infections in neonatal intensive care unit. J Infect Dis 1981;144:449-459.

19 Wong NA, Hunt LP, Marlow N: Risk factors for developing neonatal septicaemia at a Malaysian hospital. J Trop Pediatr 1997;43:5458.

20 Kuruvilla AC: Neonatal septicaemia in $\mathrm{Ku}-$ wait. J Kwt Med Assoc 1980;14:225-231.

21 West PW, Al-Sawan R, Foster HA, Electricwala Q, Alex A, Panigrahi D: Speciation of presumptive viridans streptococci from early onset neonatal sepsis. J Med Microbiol 1998;47: 923-928.

22 Manroe BL, Weinberg AG, Rosenfeld CR, Browne R: The neonatal blood count in health and disease. I. Reference values for neutrophilic cells. J Pediatr 1979;95:89-98.

23 National Committee for Clinical Laboratory Standards: Performance Standards for Antimicrobial Disk Susceptibility Tests. Approved Standard M2-A6. National Committee for Clinical Laboratory Standards.Wayne, 1997.

24 Garner JS, Jarvis WR, Emori TG, Horan TC, Hughes JM: CDC definitions for nosocomial infections. Am J Infect Control 1988;16:128140.

25 Haque KN, Chagia AH, Shaheed MM: Half a decade of neonatal sepsis, Riyadh, Saudi Arabia. J Trop Pediatr 1990;36:20-23. 
26 Garcia-Prats JA, Cooper TR, Schneider VF, Stager CE, Hansen TN: Rapid detection of microorganisms in blood cultures of newborn infants utilizing automated blood culture system. Pediatrics 2000;105:523-527.

27 Pauli I Jr, Shekhawat P, Kehl S, Sasidharan P: Early detection of bacteremia in the neonatal intensive care unit using the new BACTEC system. J Perinatol 1999;19:127-131.

28 Bennet R, Eriksson M, Zetterstrom R: Increasing incidence of neonatal septicaemia: Causative organisms and predisposing risk factors. Acta Paediatr Scand 1981;70:207-210.

29 Khan IA, Akram DS: Neonatal sepsis: Etiological study. J Pak Med Assoc 1987;37:327-330.
30 Monga K, Fernandez A, Deodhar L: Changing bacteriological patterns in neonatal septicaemia. Indian J Pediatr 1986;53:505-508.

31 Chugh K, Aggarwal BB, Kaul VK, Arya SC: Bacteriological profile of neonatal septicaemia. Indian J Pediatr 1988;55:961-965.

32 Polin RA, St Geme JW 3rd: Neonatal sepsis. Adv Pediatr Infect Dis 1992; 7:25-61.

33 Ohlsson A, Bailey T, Takieddine F: Changing etiology and outcome of neonatal septicaemia in Riyadh, Saudi Arabia. Acta Pediatr Scand 1986;75:540-544.

34 Vesikari T, Isolauri E, Tuppurainen N, Renlund M, Koivisto M, Janas M, Ikonen RS, Kero P, Heinonen K, Nyman R, et al: Neonatal septicaemia in Finland 1981-1985: Predominance of group B streptococcal infections with very early onset. Acta Paediatr Scand 1989;78: $44-50$.
35 Koutouby A, Habibullah J: Neonatal sepsis in Dubai, United Arab Emirates. J Trop Pediatr 1995;41:177-180.

36 Greenberg D, Shinwell ES, Yagupsky P, Greenberg S, Leibovitz E, Mazor M, Dagan R: A prospective study of neonatal sepsis and meningitis in Southern Israel. Pediatr Infect Dis J 1997; 16:768-773.

37 Boyer KM, Gadzala CA, Burd LI, Fisher DE, Paton JB, Gotoff SP: Selective intrapartum chemoprophylaxis of neonatal group B streptococcal early-onset disease. I. Epidemiologic rationale. J Infect Dis 1983;148:795-801. 